\title{
Nurse Practitioner-Physician Comanagement: A Theoretical Model to Alleviate Primary Care Strain
}

\author{
Allison A. Norful, RN, PbD, \\ ANP-BC $C^{1,2}$ \\ Krystyna de Jacd, MSN, MPbil, \\ PHMNP-BC ${ }^{1}$ \\ Richard Carlino, MD, FAAFP \\ Lusine Poghosyan, RN, MPH, PbD, \\ FAAN $^{1}$ \\ 'Columbia University School of Nursing, \\ New York, New York \\ ${ }^{2}$ Columbia University Medical Center \\ Irving Institute for Clinical and Transla- \\ tional Research, New York, New York
}

${ }^{3}$ Mosholu Medical Group, Bronx, New York

Conflicts of interest: authors report none.

\section{CORRESPONDING AUTHOR}

Allison A. Norful, RN, PhD, ANP-BC Columbia University School of Nursing Columbia University Medical Center Irving Institute for Clinical and Translational Research 630 W. 168th St, Mail Code 6 New York, NY 10032 aan2139@cumc.columbia.edu

\begin{abstract}
PURPOSE Various models of care delivery have been investigated to meet the increasing demands in primary care. One proposed model is comanagement of patients by more than 1 primary care clinician. Comanagement has been investigated in acute care with surgical teams and in outpatient settings with primary care physicians and specialists. Because nurse practitioners are increasingly managing patient care as independent clinicians, our study objective was to propose a model of nurse practitioner-physician comanagement.
\end{abstract}

METHODS We conducted a literature search using the following key words: comanagement; primary care; nurse practitioner OR advanced practice nurse. From 156 studies, we extracted information about nurse practitioner-physician comanagement antecedents, attributes, and consequences. A systematic review of the findings helped determine effects of nurse practitioner-physician comanagement on patient care. Then, we performed 26 interviews with nurse practitioners and physicians to obtain their perspectives on nurse practitioner-physician comanagement. Results were compiled to create our conceptual nurse practitioner-physician comanagement model.

RESULTS Our model of nurse practitioner-physician comanagement has 3 elements: effective communication; mutual respect and trust; and clinical alignment/ shared philosophy of care. Interviews indicated that successful comanagement can alleviate individual workload, prevent burnout, improve patient care quality, and lead to increased patient access to care. Legal and organizational barriers, however, inhibit the ability of nurse practitioners to practice autonomously or with equal care management resources as primary care physicians.

CONCLUSIONS Future research should focus on developing instruments to measure and further assess nurse practitioner-physician comanagement in the primary care practice setting.

Ann Fam Med 2018;16:250-256. https://doi.org/10.1370/afm.2230

\section{INTRODUCTION}

W

ith imminent staffing shortages in the health care profession and an increase in the volume of patients seeking primary care services, patient loads are increasing rapidly, thus making it dif-

ficult for a single primary care professional to manage all patient care needs effectively and efficiently. ${ }^{1-4}$ Therefore, policy makers are calling for new primary care delivery models to meet the increased demands for care, especially due to patients with multiple comorbidities requiring more complex primary care visits. Different models of care delivery have been proposed, including team-based care, yet these models often have variability in task allocation and professional roles. ${ }^{5}$ Identifying innovative models of care delivery is increasingly important to meet these demands in primary care.

One proposed care delivery model includes having more than 1 primary care professional comanaging the same patient and sharing the workload responsibilities or care management tasks. Researchers have explored comanagement of patients by 2 physicians in primary care, ${ }^{6}$ and by a physician and a nonphysician health care professional, such as a pharmacist. ${ }^{7,8}$ 
No model, however, clearly describes the comanagement relationship between physicians and advanced practice nurses, such as nurse practitioners.

Nurse practitioners are registered nurses with advanced master's or doctoral degrees whose scope of practice usually includes diagnosis and implementation of a patient care plan, regulations regarding the scope of practice vary considerably among the states regarding the need for physician involvement to treat and prescribe. ${ }^{9}$ Policy makers, and the public, have supported the expansion of nurse practitioners into primary care, ${ }^{10}$ yet the comanagement relationship between nurse practitioners and physicians remains poorly defined. As more nurse practitioners are designated as primary care clinicians and practice independent of physician oversight, a closer look at what defines successful nurse practitioner-physician comanagement is warranted. The purpose of this article is to present a theoretical model of nurse practitionerphysician comanagement in primary care.

\section{Definition of Comanagement}

We define "comanagement" as 2 primary care professionals (a nurse practitioner and a physician) jointly sharing the responsibility of all tasks needed to manage the health care of the same patient. These tasks may include patient visits, such as for acute illness or chronic disease management; pharmacologic management, such as medication refills; diagnostic testing; patient education, in terms of disease prevention or risk reduction; and patient follow-up, such as interpretation of laboratory values and making external patient referrals based on test results. Comanagement also includes sharing the administrative workload related to care coordination, completing paperwork such as disability or employment documents, and responding to patient or caregiver phone calls.

\section{History of Comanagement Model in Health Care}

One of the first studies to examine comanagement in health care was a large retrospective cohort study about orthopedic surgery. ${ }^{11}$ This study examined the effects of a surgeon and primary care physician comanaging the same patient, and results showed positive associations between comanagement and shorter hospital stays and fewer inpatient deaths. Further, comanagement has increasingly become a common practice across acute care organizations, and comanagement agreements have been implemented between surgeons and other health care professionals. ${ }^{12}$ These agreements clearly lay out responsibilities of each party, communication methods and frequency, and specific guidelines on resolution of disagreements. In the outpatient setting, researchers have focused mainly on comanagement by specialists and primary care physicians, or by pharmacists and physicians. ${ }^{7,13,14}$ These studies showed that comanagement yields optimal clinical outcomes, such as achieving blood pressure control. No published literature, however, has assessed the effects of nurse practitioner-physician comanagement.

\section{Similar Terms}

Terms such as teamwork and collaboration are often used interchangeably with comanagement. "Teamwork," however, is defined as a group of people working interdependently to achieve a common goal ${ }^{9}$ and "collaboration" is defined as 2 clinicians consulting with each other and working concurrently by sharing knowledge and expertise to achieve optimal patient care. ${ }^{15}$ Evidence is clear about the benefits of team-based and collaborative care, ${ }^{16}$ yet researchers have concluded that evidence is lacking about comanagement approaches to care. ${ }^{17}$

Team-based care and collaborative care with nurse practitioners often involve a hierarchy with team members aligned in a vertical organizational structure based on profession or role. Vertical hierarchy in an organization influences decision making and subsequently may impede communication or increase mistrust among team members from various professions. ${ }^{18}$ In contrast, comanagement involves a horizontal organizational structure. Clinicians may comanage across teams in a manner similar to a primary care physician and a cardiologist comanaging the same patient. These 2 physicians work within their own teams within their practices, but overlap horizontally to comanage the same patient. Within the same team, an independent nurse practitioner may comanage the same patient with a physician, in the same practice, based on the urgency or complexity of a patient's needs. While research has found evidence of the attributes of teamwork, including honesty, discipline, creativity, humility, and curiosity, ${ }^{19}$ the literature fails to capture the attributes of comanagement between nurse practitioners and physicians.

\section{METHODS}

We built our model from the collective findings of 3 studies. First, using Walker and Avant's method for conceptual analysis, ${ }^{20}$ we conducted a literature search in 5 electronic databases (Ovid Medline, CINAHL, PubMed, Cochrane Review, and EMBASE) using the following key words: comanagement; primary care; nurse practitioner OR advanced practice nurse. A total of 156 studies were reviewed. We extracted information about nurse practitioner-physician comanagement antecedents, relationships, defining attributes, and consequences. Next, using the 
PRISMA framework, ${ }^{21}$ a systematic review was conducted to determine the effects of nurse practitioner-physician comanagement and found an increase in primary care clinician adherence to recommended care guidelines and improved clinical patient outcomes. ${ }^{22}$ Third, we performed in-person qualitative interviews with nurse practitioners and physicians to obtain their perspectives on nurse practitioner-physician comanagement including the willingness of primary care professionals to comanage care, descriptions of the dimensions of comanagement, and how nurse practitioner-physician comanagement affects patient care. Twenty-six interviews were conducted until data saturation was reached and no new information was emerging from the interviews. ${ }^{22,23}$ Results of all 3 studies were triangulated to build the conceptual nurse practitioner-physician comanagement model.

\section{Theoretical Underpinnings}

Our approach to investigating nurse practitioner-physician comanagement was guided by the theoretical underpinnings of Donabedian's quality of care mode ${ }^{24}$ (Figure 1). This model provided us with a framework to evaluate the quality of comanagement. Two researchers met weekly to discuss the findings from the 3 studies and extract information about each of the 3 dimensions of quality of care (structure, process, and outcome). First, the researchers obtained information about comanagement structure, which involved the organizational and clinician resources or policies that needed to be in place for nurse practitioners and physicians to comanage the same primary care patient Next, we evaluated process, that is, how comanagement was being practiced, what interactions were necessary, and the interprofessional relationships between nurse practitioners and physicians. Finally, we evaluated outcomes, which included the results of our systematic review and the reported perspectives of the primary care professionals in our qualitative study.

\section{RESULTS}

\section{Antecedents of Nurse Practitioner-Physician Comanagement}

The primary antecedent for effective nurse practitioner-physician comanagement is nurse practitioner autonomy. Various policy bodies regulate nurse practitioner scope of practice and nurse practitioner licensure, leading to a wide variablity. ${ }^{9}$ In addition to national or state-based legislation that defines the nurse practitioner scope of practice, nurse practitioner responsibilities are often determined by organizational policy. ${ }^{25}$ Despite the adoption of laws that allow nurse practitioners to practice independently of physician oversight, organizational or facility policy may inhibit and restrict a nurse practitioner-physician comanagement model. These restrictions are especially salient in the primary care clinics that adopt a physician-led hierarchical infrastructure in which the physician has the final decision-making authority. In this case, the nurse practitioners do not comanage the patient care but exercise a limited role. Further, organizational climate, and the culture of organizations, heavily influenced by organizational management, often do not identify and/ or do not accept nurse practitioners as primary care clinicians ${ }^{26}$ In this situation, the organization does not provide the same resources to nurse practitioners as they do physicians. ${ }^{27}$ These resources include support staff, such as medical assistant help, enough examination rooms for patient visits, involvement on decisionmaking committees, and availability of learning opportunities. ${ }^{23,28}$ Our model focused specifically on comanagement in which nurse practitioners and physicians were viewed equally as primary care clinicians, shared equal responsibility for primary care patient management, and were provided with equal resources.

\section{Vital Attributes}

Effective nurse practitioner-physician comanagement has 3 vital attributes: (1) effective communication ${ }_{i}(2)$ mutual respect and trust; and (3) clinical alignment, also known as a shared philosophy of care (Figure 2).

\section{Effective Communication}

Effective communication is a 2-way process in which primary care professionals send a message that is easily understood by the receiving party to prevent misunderstanding and to save time. Comanagement communication is essential for developing the patient care plan, managing a change in patient health status, individualizing patient goals, and delineating each primary care clinician's role in the care plan as part of coordinating 


\section{Figure 2. Nurse practitioner-physician comanagement.}

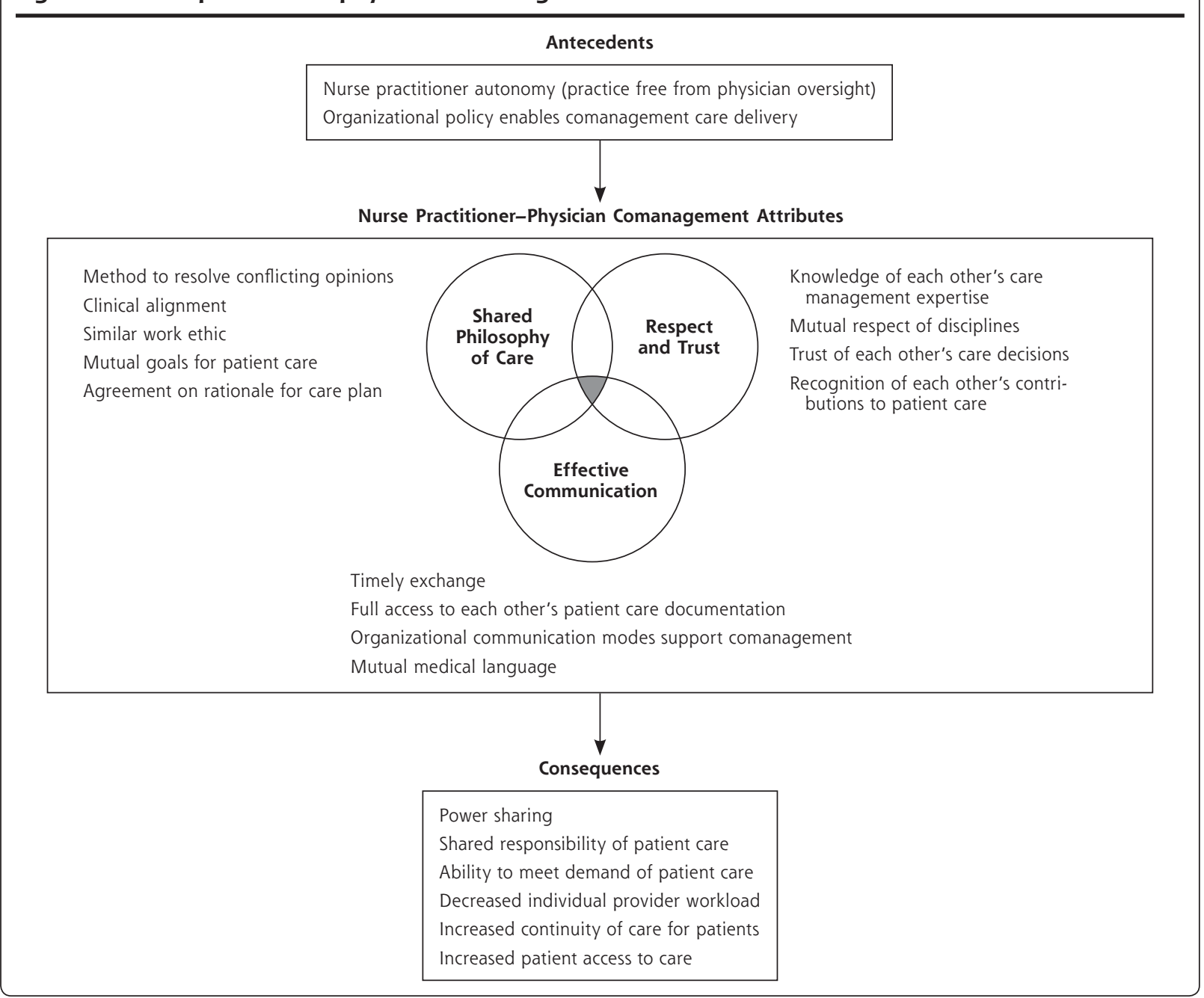

patient care. ${ }^{29}$ When the nurse practitioner and physician who are comanaging a patient do not have direct contact with each during their daily activities, the use of secure messaging through an electronic health record (EHR) or telephone calls are the most frequent form of communication. ${ }^{23}$ Some EHR systems, however, have been found to inhibit communication because the nurse practitioner and physician documentation is located in separate locations within the patient chart, thus preventing them from seeing each other's notes. ${ }^{23}$ The setting size and space often influence the type of communication used, with smaller settings using more informal modes of communication, such as text messages. ${ }^{30}$ Comanagement communication must be performed in a timely manner that is dependent on the patient needs, such as a change in patient acuity level. The communication needs to be reciprocal with equal sharing of ideas, new patient information, and feedback necessary to improve quality of care. ${ }^{31}$

\section{Mutual Respect and Trust}

Respect and trust among nurse practitioners and physicians is the second critical element of comanagement. This attribute increases over time as physicians and nurse practitioners work together longer ${ }^{32}$; developing reciprocal trust and respect of each other's role in care delivery can take up to 6 months. ${ }^{30}$ By gaining trust, physicians are less likely to feel that they need to supervise or "double-check" the work of the nurse practitioner, thereby reducing redundancy of documentation and diagnostic testing.

Traditionally, some physicians view nurse practitioners as having an inferior role in primary care. This viewpoint inhibits nurse practitioners from working to their full potential and can create mistrust or resentment. The physician must have an understanding of the education, training, and scope of practice for nurse practitioners to build trust during allocation of tasks and responsibilities. ${ }^{25}$ The optimal combination of 
nurse practitioners' and physicians' knowledge, culture, and disciplines has the potential to positively contribute to the quality of patient care.

\section{Shared Philosophy of Care}

Physicians and nurse practitioners that we interviewed agreed that each primary care professional must have complementary practice styles that are congruent to mutual goals for patient care, such as a shared philosophy or having a clinical alignment in their patient care plan. ${ }^{31}$ This shared philosophy includes approaches to care management. Variability of approaches challenges nurse practitioner-physician comanagement. For example, one clinician may opt to treat mental illness in primary care while the other clinician prefers referral to a specialist. Other examples include when to prescribe an antibiotic or when to discontinue a patientspecific treatment, such as pain management.

In the event of disagreement between primary care clinicians regarding care decisions, discussion is vital. However, a mutually agreed-upon protocol for conflict resolution must be in place ahead of time to determine who makes the final care management decision. This protocol may vary by organizational policy or practice setting. Clinical alignment also involves a similar work ethic, such as time management styles. Without a similar work ethic, the workload may become unbalanced and weighted toward 1 of the clinicians, potentially leading to clinician burnout and increased strain. One of the primary care professionals having a higher volume of daily patients than the other clinician may lead to resentment, which may threaten mutual respect and trust or communication, with the potential of indirectly affecting patient care.

\section{Consequences of Comanagement}

At the level of the primary care professional, the presence of all 3 attributes of the model leads to clinician cohesion. The stronger comanagement is, the greater the potential for beneficial patient, clinician, and practice outcomes. ${ }^{22}$ One finding of our interviews was that effective nurse practitioner-physician comanagement alleviated individual clinician workload and the strain to complete all recommended clinical care and administrative tasks singlehandedly. A reduction of primary care professional workload subsequently prevents clinician strain, burnout, and fatigue, especially with increased patient complexity. Nurse practitionerphysician comanagement also enables interdisciplinary collaboration between nursing and medicine, and better care results from combining the experience and expertise of clinicians from each discipline. Interdisciplinary collaboration also promotes morale among team members and leads to effective and efficient outcomes. ${ }^{33,34}$
Nurse practitioner-physician comanagement was also found to increase patient access to care and promote continuity of care because patients have 2 clinicians familiar with their history and care needs..$^{29,35}$ Longevity of patient and primary care professional interactions is often described as a core value of highquality primary care. ${ }^{36,37}$ Further, fewer restrictions on the scope of practice for nurse practitioners is associated with an increase in the number of nurse practitioners practicing in rural or medically underserved populations. ${ }^{38}$ Nurse practitioner-physician comanagement in rural or medically underserved populations allows primary care physicians to free time up for additional appointments, as well as provide patients with more one-on-one time during patient visits to address individual patients' needs. ${ }^{3}$

\section{DISCUSSION}

More nurse practitioners are practicing as independent primary care professionals, and developing innovative approaches to integrate nurse practitioners and physicians within and across team-based care models is important. This article presents a theoretical model of nurse practitioner-physician comanagement, including the vital attributes of effective communication, mutual respect and trust, and shared philosophy of care.

This novel theoretical understanding has several potential uses. First, use of this model can help create organizational policies needed to ensure the success of nurse practitioner-physician comanagement. When administrators, clinicians, and policy makers promote effective comanagement, individual clinician workload is reduced, thus preventing clinician strain, burnout, and fatigue, especially with increased patient complexity. ${ }^{23}$ Use of this model also enables increased collaboration among clinicians who discuss and coordinate the complex needs of patients, thereby providing higher quality of care. ${ }^{34,39}$ Effective nurse practitioner-physician comanagement also has the potential to increase access to care because patients have 2 primary care professionals familiar with their needs and plan of care, thus promoting a continuity of care. If 1 clinician is unavailable, the other can see the patient, preventing a gap in access to care. By sharing the workload, nurse practitioner-physician comanagement can lead to time for additional appointments and/or more one-on-one individualized attention to patient needs. We recommend efforts toward interdisciplinary education within academic institutions so that nurse practitioners and physicians gain knowledge of each other's disciplines early on and learn strategies to comanage patient care given the complexities of primary care delivery and the identified strengths of each discipline. 
Attention to individualized patient care is especially important as reimbursement mechanisms shift from volume-based to value-based care and provider payments are reliant on achieving targeted quality outcomes. ${ }^{40,41}$ The combination of nurse practitioner and physician expertise in comanagement can help to ensure the highest quality of care. Several studies included in our systematic review demonstrated a significant difference in guideline adherence in favor of nurse practitioners and physicians comanagement of the same patient. ${ }^{22}$ Furthermore, evidence shows that nurse practitioners in primary care professional roles have equivalent or superior patient outcomes and are potentially cost saving. ${ }^{42}$ This finding suggests the potential of nurse practitioner-physician comanagement to be more cost effective than 2 physicians comanaging care. More cost-effective studies about nurse practitionerphysician comanagement are warranted.

Lastly, despite the increasing numbers of nurse practitioners and physicians who are already comanaging in practice, a substantial gap in the literature remains about how organizations should design comanagement models. More evidence is needed about which care delivery models are the most efficient and effective in primary care. Nurse practitioner-physician comanagement demonstrates promise to alleviate some of the primary care strain, but more research is needed to produce empirical and generalizable evidence about its impact on clinical, cost, and organizational outcomes. Our theoretical model provides health services researchers with knowledge to operationalize nurse practitioner-physician comanagement in future studies.

A survey instrument is currently being developed from this theoretical model and tested psychometrically to enable measurement of nurse practitionerphysician comanagement in practice and research settings. This survey instrument, once validated, will provide primary care physicians, practice managers, policy makers, and researchers the ability to further investigate nurse practitioner-physician comanagement and its impact on patient or practice outcomes.

The 3 vital attributes from our nurse practitionerphysician comanagement model-effective communication, mutual respect and trust, and a shared philosophy of care-cannot exist without the presence of legal and organizational policies that recognize nurse practitioners as autonomous primary care clinicians. Further, effective nurse practitioner-physician comanagement requires adequate organizational resources and the willingness of nurse practitioners and physicians to comanage. Opposing opinions about the autonomy of nurse practitioners and the drive for physician-led hierarchical infrastructures have pre- vented autonomous practice of nurse practitioners in primary care. ${ }^{43}$ As long as such limitations exist, the effective comanagement care model cannot be fully investigated or implemented. We recommend empirical measurement of nurse practitioner-physician comanagement for future research.

To read or post commentaries in response to this article, see it online at http://www.AnnFamMed.org/content/16/3/250.

Key words: primary care; nurse practitioner; comanagement; theory

Submitted July 5, 2017; submitted, revised, November 1, 2017; accepted November 30, 2017.

Funding support: This study was supported by the National Institute of Nursing Research (T32 NR014205) and the National Center for Advancing Translational Sciences, National Institutes of Health (TL1TR001875).

Disclaimer: The content is solely the responsibility of the authors and does not necessarily represent the official views of the $\mathrm{NIH}$.

Previous presentations: This paper was presented at the Academy Health Annual Research Meeting; June 25-27, 2017; New Orleans, Louisiana, and the 2016 Eastern Nurses Research Society Annual Meeting; April 13-15, 2016; Pittsburgh, Pennsylvania.

\section{References}

1. Wu SY, Green A. Projection of Chronic Illness Prevalence and Cost Inflation. Santa Monica, CA: RAND Corporation; 2000.

2. World Health Organization (WHO). Noncommunicable diseases: progress monitor 2015. http://apps.who.int/iris/bitstr eam/10665/184688/1/9789241509459_eng.pdf?ua=1. Published 2015. Accessed Jul 3, 2017

3. Yarnall KS, Østbye T, Krause KM, Pollak KI, Gradison M, Michener JL. Family physicians as team leaders: "time" to share the care. Prev Chronic Dis. 2009;6(2):A59.

4. World Health Organization. (WHO). World health statstics: monitoring health for the sustainable development goals. http://apps.who. int/iris/bitstream/10665/255336/1/9789241565486-eng.pdf?ua=15. Published 2017. Accessed Oct 15, 2017.

5. Tiedeman ME, Lookinland S. Traditional models of care delivery: what have we learned? J Nurs Adm. 2004;34(6):291-297.

6. Rose DE, Tisnado DM, Tao ML, et al. Prevalence, predictors, and patient outcomes associated with physician co-management: findings from the Los Angeles Women's Health Study. Health Serv Res. 2012;47(3 pt 1):1091-1116.

7. Von Muenster SJ, Carter BL, Weber CA, et al. Description of pharmacist interventions during physician-pharmacist co-management of hypertension. Pharm World Sci. 2008;30(1):128-135.

8. Chen Z, Ernst ME, Ardery G, Xu Y, Carter BL. Physician-pharmacist co-management and 24-hour blood pressure control. J Clin Hypertens (Greenwich). 2013;15(5):337-343.

9. Brush JE Jr, Handberg EM, Biga C, et al. 2015 ACC health policy statement on cardiovascular team-based care and the role of advanced practice providers. J Am Coll Cardiol. 2015;65(19): 2118-2136.

10. American College of Physicians. Nurse practitioners in primary care: a policy monograph of the American College of Physicians. https://www.acponline.org/acp_policy/policies/nursepractitioners_ pc_2009.pdf. Published 2009. Accessed Jul 3, 2017. 
11. Hinami K, Feinglass J, Ferranti DE, Williams MV. Potential role of comanagement in "rescue" of surgical patients. Am J Manag Care. 2011;17(9):e333-e339.

12. Cheng HQ. Comanagement: who's in charge? AHRQ, Patient Safety Network web site. https://psnet.ahrq.gov/webmm/case/271/ comanagement-whos-in-charge. Published Jun 2012. Accessed Jul 3, 2017.

13. Weber CA, Ernst ME, Sezate GS, Zheng S, Carter BL. Pharmacistphysician comanagement of hypertension and reduction in 24-hour ambulatory blood pressures. Arch Intern Med. 2010;170(18): 1634-1639.

14. Bowman BT, Kleiner A, Bolton WK. Comanagement of diabetic kidney disease by the primary care provider and nephrologist. Med Clin North Am. 2013;97(1):157-173.

15. Bridges S. Exploration of the concept of collaboration within the context of nurse practitioner-physician collaborative practice. J Am Assoc Nurse Pract. 2014;26(7):402-410.

16. Wen J, Schulman KA. Can team-based care improve patient satisfaction? A systematic review of randomized controlled trials. PLoS One. 2014;9(7):e100603.

17. Harrington RA, Heidenreich PA. Team-based care and quality: a move toward evidence-based policy. J Am Coll Cardiol. 2015;66(16): 1813-1815.

18. Armstrong JH. Leadership and team-based care. Virtual Mentor. 2013;15(6):534-537.

19. Mitchell P, Wynia M, Golden R, McNellis B, Oku S, Webb CE, et al. Discussion paper: core principles $\&$ values of effective team-based health care. https://www.nationalahec.org/pdfs/vsrt-team-basedcare-principles-values.pdf. Published Oct 2012. Accessed Oct 2017.

20. Walker L, Avant K. Concept analysis. In: Strategies for Theory Construction in Nursing. New York, NY: Pearson; 2005:37-54.

21. Moher D, Liberati A, Tetzlaff J, Altman DG; PRISMA Group. Preferred reporting items for systematic reviews and meta-analyses: the PRISMA statement. Ann Intern Med. 2009;151(4):264-269, W64.

22. Norful AA, Swords K, Marichal M, Cho H, Poghosyan L. Nurse practitioner-physician comanagement of primary care patients: the promise of a new delivery care model to improve quality of care. [published online ahead of print April 25, 2017]. Health Care Manage Rev.

23. Norful AA. Nurse Practitioner-Physician Co-management of Primary Care Patient Panels: Impact, Perspective, and Measurement toward a New Delivery Care Model [dissertation]. New York, NY: Columbia University; 2017.

24. Donabedian A. The quality of care. How can it be assessed? JAMA. 1988;260(12):1743-1748.

25. Schadewaldt V, McInnes E, Hiller JE, Gardner A. Views and experiences of nurse practitioners and medical practitioners with collaborative practice in primary health care - an integrative review. $B M C$ Fam Pract. 2013;14(1):132.

26. Kilpatrick K, Lavoie-Tremblay M, Ritchie JA, Lamothe L. Advanced practice nursing, health care teams, and perceptions of team effectiveness. J Trauma Nurs. 2014;21(6):291-299.
27. Hall P. Interprofessional teamwork: professional cultures as barriers. J Interprof Care. 2005;19(Suppl 1):188-196.

28. Almost J, Laschinger HK. Workplace empowerment, collaborative work relationships, and job strain in nurse practitioners. J Am Acad Nurse Pract. 2002;14(9):408-420.

29. Way D, Jones L, Busing N. Implementation strategies: "collaboration in primary care-family doctors $\&$ nurse practitioners delivering shared care": discussion paper written for the Ontario College of Family Physicians. http://citeseerx.ist.psu.edu/viewdoc/download?do $\mathrm{i}=10.1 \cdot 1.458 .383 \mathrm{Erep}=$ rep1Etype $=$ pdf. Published May 18, 2000. Accessed Oct 1, 2017.

30. Brault I, Kilpatrick K, D'Amour D, et al. Role clarification processes for better integration of nurse practitioners into primary healthcare teams: a multiple-case study. Nurs Res Pract. 2014;2014:170514.

31. Hallas DM, Butz A, Gitterman B. Attitudes and beliefs for effective pediatric nurse practitioner and physician collaboration. J Pediatr Health Care. 2004;18(2):77-86.

32. Schadewaldt V, McInnes E, Hiller JE, Gardner A. Investigating characteristics of collaboration between nurse practitioners and medical practitioners in primary healthcare: a mixed methods multiple case study protocol. J Adv Nurs. 2014;70(5):1184-1193.

33. Al Sayah F, Szafran O, Robertson S, Bell NR, Williams B. Nursing perspectives on factors influencing interdisciplinary teamwork in the Canadian primary care setting. J Clin Nurs. 2014;23(19-20): 2968-2979.

34. Grumbach K, Bodenheimer T. Can health care teams improve primary care practice? JAMA. 2004;291(10):1246-1251.

35. Freeman GK, Olesen F, Hjortdahl P. Continuity of care: an essential element of modern general practice? Fam Pract. 2003;20(6): 623-627.

36. Freeman G, Shepperd S, Robinson I, et al. Continuity of care. Report of a Scoping Exercise for the National Co-ordinating Centre for NHS Service Delivery and Organisation R \& D (NCCSDO). London, England:NCCSDO; 2001.

37. Delva D, Kerr J, Schultz K. Continuity of care: differing conceptions and values. Can Fam Physician. 2011;57(8):915-921.

38. Xue $Y, Y e Z$, Brewer C, Spetz J. Impact of state nurse practitioner scope-of-practice regulation on health care delivery: systematic review. Nurs Outlook. 2016;64(1):71-85.

39. Carless SA, De Paola C. The measurement of cohesion in work teams. Small Group Res. 2000;31(1):71-88.

40. Asher AL, Devin CJ, Mroz T, Fehlings M, Parker SL, McGirt MJ. Clinical registries and evidence-based care pathways: raising the bar for meaningful measurement and delivery of value-based care. Spine (Phila Pa 1976). 2014;39(22)(Suppl 1):S136-S138.

41. Burwell SM. Setting value-based payment goals-HHS efforts to improve U.S. health care. N Engl J Med. 2015;372(10):897-899.

42. Martin-Misener R, Harbman P, Donald F, et al. Cost-effectiveness of nurse practitioners in primary and specialised ambulatory care: systematic review. BMJ Open. 2015;5(6):e007167.

43. Mullinix C, Bucholtz DP. Role and quality of nurse practitioner practice: a policy issue. Nurs Outlook. 2009;57(2):93-98. 